УДК 661.832.321.065..5

\title{
B.3. Пойлов
}

Пермский национальный исследовательский политехнический университет, Пермь, Россия

\section{ЗАКОНОМЕРНОСТИ МАССОВОЙ ПОЛИТЕРМИЧЕСКОЙ КРИСТАЛЛИЗАЦИИ ХЛОРИДА КАЛИЯ}

Получение кристаллических осадков с заданным гранулометрическим составом является важной, но непростой задачей многих химических технологий. Сложность решения проблемы заключается в том, что массовая кристаллизация веществ сопровождается одновременным протеканием процессов первичного и вторичного зародышеобразования, агрегаџии и роста кристаллов, их разрушения, перекристаллизации. Получаемый при этом кристаллический осадок имеет характеристики, отражающие результаты превалирующего воздействия одного или нескольких из указанных процессов. Целью данной работьл являлось установление закономерностей формирования осадка хлорида калия с определенным гранулометрическим составом в процессе массовой политермической кристаллизаџии. С использованием лазерного анализатора размеров частии, позволяющего проводить измерения дисперсных характеристик осадков непосредственно в прочессе их образования, исследованы закономерности формирования частии $\mathrm{KCl}$ в прочессе массовой политермической кристаллизачии в аппарате периодчческого действия. Исследования кристаллизации проводили в кристаллизаторе объемом 1 л, снабженном водяной рубашкой и пропеллерной мешалкой с электронной системой задания и контроля скорости перемешивания 600 об/мин и охлаждения, обеспечивающей режим витания кристаллов и охлаждение с 85 до $28^{\circ} \mathrm{C}$ по экспоненциальной зависимости $T=\exp (4,24981-0,0148 \mathrm{t})$, где $T$ - температура раствора, ${ }^{o} C ;$ - $t$ - дительность процесса, $c$. Выявлен затухающий характер колебаний скорости генераџии частии. Установлена значительная роль агломерачионного роста частиц. Влияние такого механизма формирования частии по мере кристаллизачии постепенно снижается за счет механического разрушения агломератов и вторичного зародьишеобразования. Предложены эмпирические уравнения, описывающие кинетику процессов образования и роста частии хлорида калия при политермической кристаллизации. Введение в чистый кристаллизуемый раствор небольших количеств солянокисльх аминов $\left(\mathrm{C}_{16} \mathrm{H}_{33}-\mathrm{C}_{18} \mathrm{H}_{37}\right) \mathrm{NH}_{3} \mathrm{Cl}$ приводит к снижению скорости первичной нуклеачии, но не подав- 
ляет агломерационный рост частии, увеличивает дисперсии размеров частии в начале прочесса политермической кристаллизачии.

Ключевые слова: политермическая кристаллизация, хлорид калия, кинетика зародышеобразования, дифференцииальные кривые числового распределения частиц по размерам, эволючия.

\section{V.Z. Pojlov}

Perm National Research Polytechnic University, Perm, Russian Federation

\section{PATTERNS OF POTASSIUM CHLORIDE MASS POLYTERMIC CRYSTALLIZATION}

Production of crystalline precipitation with specific particle size distribution is an important but difficult task for many chemical technologies. Complexity of this problem is due to the fact that mass crystallization of substances accompanied by simultaneous primary and secondary nucleation, crystal growth and aggregation, their destruction and recrystallization. Obtained crystalline precipitate has characteristics which are formed by influence of the one or more major processes. The aim of this work was to establish the main principles of potassium chloride with a certain particle size distribution precipitate formation during the process of mass polythermal crystallization. Main principles of KCI formation in mass polythermal crystallization process are studied by using laser particle size analyzer. Is allows to measure dispersed precipitation characteristics directly during their formation. A significant role of the agglomeration of particle growth is shown. The impact of such a particle formation mechanism as crystallization gradually decreases due to mechanical break up agglomerates and secondary nucleation. Empirical equations describing the kinetics of the formation and growth of potassium chloride particles at polythermal crystallization are proposed. Introducing of small quantities of amines $\left(\mathrm{C}_{16} \mathrm{H}_{33}-\right.$ $\left.\mathrm{C}_{18} \mathrm{H}_{37}\right) \mathrm{NH}_{3} \mathrm{Cl}$ into the pure crystallizable hydrochloric solution leads to reduction of the primary nucleation rate, but does not inhibit growth of particle agglomeration, particle size of the dispersion increases in the beginning of the crystallization process polythermal.

Keywords: polytermic crystallization, potassium chloride, $\mathrm{nu-}$ cleation kinetics, differential curves, granulometric composition, size distribution, evolution.

Получение кристаллических осадков с заданным гранулометрическим составом является важной, но непростой задачей многих химических технологий. Сложность решения проблемы заключается в том, что массовая кристаллизация веществ сопровождается одновременным 
протеканием процессов первичного и вторичного зародышеобразования, агрегации и роста кристаллов, их разрушения, перекристаллизации [1-3]. Получаемый при этом кристаллический осадок имеет характеристики, отражающие результаты превалирующего воздействия одного или нескольких из указанных процессов. При существенном вкладе первичного зародышеобразования получаемый осадок имеет частицы малого размера с узким мономодальным распределением частиц по размерам [3, 4]. Процессы роста и агломерации частиц способствуют укрупнению кристаллов (в виде агрегатов) с получением более широкого распределения частиц по размерам [5]. Проявление процессов вторичного зародышеобразования и механического разрушения, как правило, сопровождается формированием осадков с полимодальным распределением частиц по размерам. При этом кристаллы осадка могут иметь неправильную или неизометричную форму, в виде осколков. Процесс перекристаллизации чаще всего ведет к уменьшению содержания в осадке мелкодисперсных фракций, повышению степени изометричности кристаллов $[6,7]$. Управление каждым из указанных процессов требует знаний особенностей их протекания и воздействия этих процессов на конечный гранулометрический состав кристаллизата $[8,9]$.

С развитием современной техники лазерной дифракции в последние годы возросли возможности экспериментального исследования процесса массовой кристаллизации веществ из растворов с прямым измерением параметров дисперсности кристаллических частиц. Постановка таких экспериментов позволяет расширить и углубить знания о закономерностях протекания сложных в изучении процессов массовой кристаллизации и создать в перспективе теоретические основы для технологий получения кристаллических продуктов с заданными свойствами. Целью данной работы являлось установление закономерностей формирования осадка хлорида калия с определенным гранулометрическим составом в процессе массовой политермической кристаллизации.

\section{Экспериментальная часть}

Исследования кристаллизации хлорида калия проводили в кристаллизаторе объемом 1 л, снабженном водяной рубашкой и пропеллерной мешалкой с электронной системой задания и контроля скорости перемешивания при постоянной скорости перемешивания 600 об/мин, обеспечивающей режим витания кристаллов. В рубашке кристаллизатора поддерживали заданную температуру с точностью $0,1^{\circ} \mathrm{C}$ с помощью 
термостата программного охлаждения фирмы «Medingen». Для измерений числа частиц, попадающих в область сканирования лазерного пучка, датчик анализатора «Partec-2000» был помещен непосредственно в кристаллизуемую среду. Данный прибор позволяет определять число частиц и их размеры. Но так как прибор производит регистрацию частиц только в плоскости фокусного расстояния, а в кристаллизаторе суспензия при перемешивании многократно протекает через плоскость сканирования, то измерения числа частиц являются относительными. Охлаждение раствора проводили по экспоненциальному режиму охлаждения. Так, охлаждение с 85 до $28{ }^{\circ} \mathrm{C}$ по экспоненциальной зависимости подчинялось уравнению

$$
T=\exp (4,24981-0,0148 t),
$$

где $T$ - температура раствора; ${ }^{\circ} \mathrm{C} ; t$ - длительность процесса, с.

Для исследований использовали отфильтрованный через плотный фильтр раствор хлорида калия, в котором фоновое число регистрируемых частиц составляло 20-25 $\mathrm{c}^{-1}$. При этом во всех опытах перед началом кристаллизации исходный раствор перегревали выше температуры насыщения на $10{ }^{\circ} \mathrm{C}$, выдерживали 10 мин при постоянной скорости перемешивания и охлаждали до температуры начала кристаллизации со скоростью 0,5 град/мин. Начало массовой кристаллизации определяли по превышению числа регистрируемых частиц выше фонового значения. Воспроизводимость данных при строго фиксированных условиях кристаллизации контролировали по числу образующихся частиц и значению температуры начала массовой кристаллизации.

Кристаллизацию КС1 проводили из чистого раствора и раствора, содержащего 0,001 \% солянокислого амина $\left(\mathrm{C}_{16} \mathrm{H}_{33}-\mathrm{C}_{18} \mathrm{H}_{37}\right) \mathrm{NH}_{3} \mathrm{Cl}$.

\section{Результаты экспериментов}

Экспериментальные данные по относительному числу генерируемых в процессе кристаллизации частиц $\mathrm{KCl}$ приведены на рис. 1 . Как видно на рис. 1, в первые 100 с процесса массовой кристаллизации наблюдается быстрое возрастание числа частиц, которое в середине процесса постепенно замедляется. Причем присутствие в кристаллизуемом растворе адсорбирующихся на кристаллах $\mathrm{KCl}$ аминов значительно снижает число образующихся частиц на всем протяжении политермической кристаллизации. Дифференцирование полученных данных по времени позволяет определить относительные скорости образования частиц $\mathrm{KCl}$ (рис. 2). На рис. 2 можно видеть, что скорость 


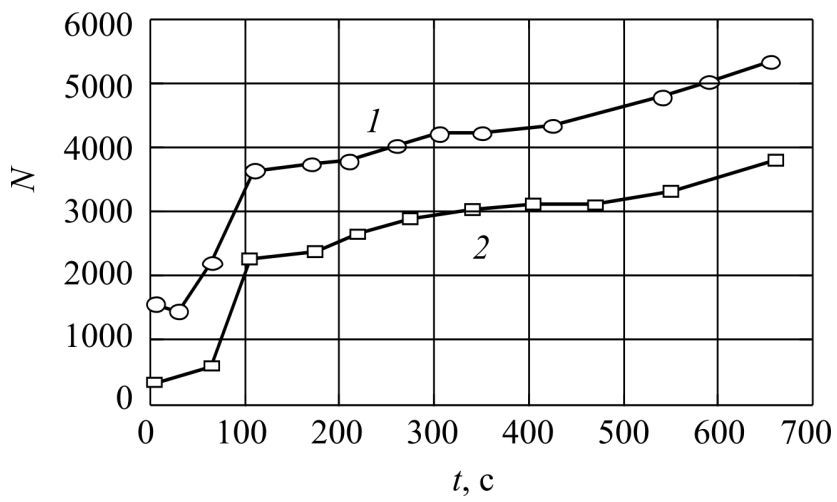

Рис. 1. Изменение числа регистрируемых частиц КС1 в процессе кристаллизации: 1 - из чистого раствора; 2 - из раствора, содержащего солянокислый амин

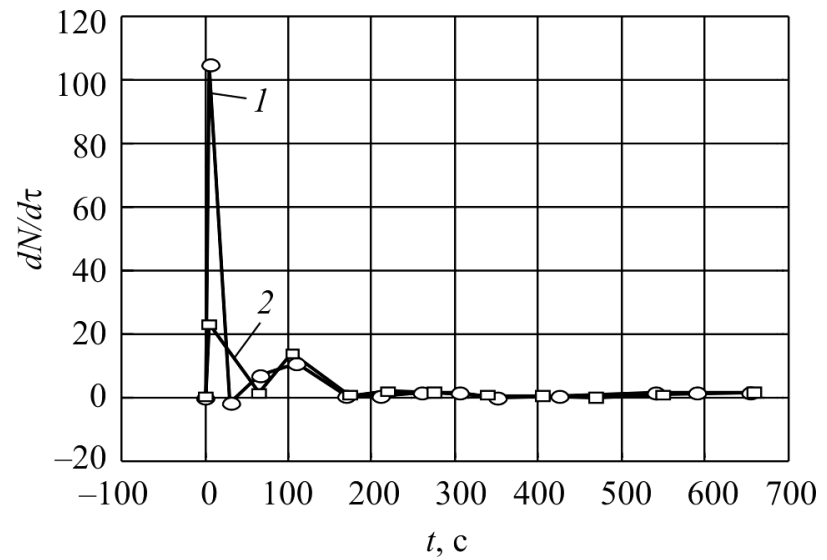

Рис. 2. Изменение относительных скоростей образования частиц хлорида калия при политермической кристаллизации: 1 - из чистого раствора; 2 - из раствора, содержащего амин

образования частиц в процессе кристаллизации имеет колебательный характер. Первую пульсацию скорости можно соотнести с первичным зародышеобразованием. Этот процесс имеет максимальную скорость, так как протекает при высокой температуре раствора, максимальном пересыщении и малой длительности снятия пересыщения. Вторая пульсация скорости, происходящая через 105-110 с, имеет величину скорости, в несколько раз меньшую, чем скорость первичной нуклеации. Последующие пульсации относительной скорости образования частиц почти на порядок ниже, чем при второй пульсации. Таким образом, скорость образования частиц в процессе политермической кристалли- 
зации с экспоненциальным режимом охлаждения в кристаллизаторе периодического действия имеет характер затухающих колебаний, причем первичная нуклеация протекает значительно интенсивнее, чем вторичная.

Известно, что на скорость образования частиц при массовой кристаллизации помимо пересыщения большое влияние оказывают величина температуры, межфазной поверхности, частота и энергия столкновений частиц [1]. Рассмотрим, как изменяется в процессе политермической кристаллизации удельная поверхность $A$ образующихся частиц $\mathrm{KCl}$ :

$$
A=\beta \int n(L) L^{2} d L
$$

где $\beta$ - фактор формы для кубических кристаллов $\mathrm{KCl}, \beta=6 ; n(L)$ - плотность распределения числа частиц по размерам $L$.

Значения величин внешней поверхности $A$ приведены на рис. 3 , где видно, что наибольшие значения удельная поверхность приобретает при $t=100$ с и далее она постепенно снижается. Отметим, что в этот же момент времени в кристаллизуемом растворе наблюдается вторая пульсация относительной скорости образования частиц (см. рис. 2), появление которой связано с интенсификацией вторичной нуклеации в результате быстрого возрастания.

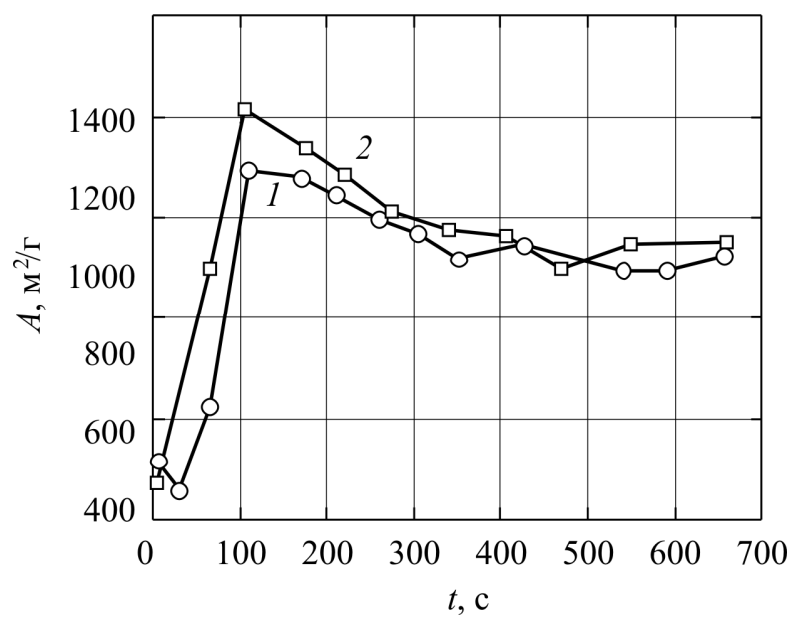

Рис. 3. Изменение удельной поверхности частиц $\mathrm{KCl}$

в процессе политермической кристаллизации:

1 - из чистого раствора; 2 - из раствора, содержащего амин 
величины удельной поверхности. Снижение величины межфазной поверхности в последующие моменты времени, а также температуры и пересыщения раствора оказывают решающее влияние на затухающий характер скорости образования частиц $\mathrm{KCl}$. В то же время возрастание числа частиц, их массы и энергии столкновений, происходящее в процессе кристаллизации и способствующее ускорению вторичной нуклеации, оказывает значительно меньшее влияние на скорость образования частиц.

Поскольку процесс образования частиц $\mathrm{KCl}$ происходит в условиях непрерывно изменяющихся температуры, пересыщения, плотности и вязкости суспензии и сопровождается параллельно протекающими стадиями роста, агломерации и разрушения частиц, кинетика которых недостаточно изучена, то интегральный процесс генерации частиц можно представить в виде эмпирических выражений, применимых в области исследованных значений скорости перемешивания и при заданном температурном режиме охлаждения. Так, приведенная на рис. 1 зависимость $N=f(t)$ описывается уравнением следующего вида:

$$
N=N_{\mathrm{o}} \exp (-k / t),
$$

где $N_{\text {o }}=5103$ и $k=47,0$ при кристаллизации из чистого раствора и $N_{\mathrm{o}}=3990$ и $k=88,5$ при кристаллизации из раствора, содержащего амин.

При подстановке выражения (1) в уравнение (3) относительное число генерируемых частиц $\mathrm{KCl}$ может быть вычислено из данных значений температуры среды в кристаллизаторе:

$$
N=N_{1} \exp (-b /(\ln T-4,24981),
$$

где $N_{1}=5103$ и $b=0,6956$ при кристаллизации из чистого раствора; $N_{1}=3990$ и $b=0,12655$ при кристаллизации из аминированного раствора.

Значительное влияние низкой концентрации аминов на скорость образования частиц проявляется в наибольшей степени в процессе первичной нуклеации (см. рис. 2). Наиболее вероятный механизм такого влияния заключается в том, что амины адсорбируются на дислокациях кристаллических зародышей и предотвращают их рост до критических размеров, снижая тем самым число зародышей критических размеров. Кроме того, адсорбируясь на стенках, мешалке кристаллизатора и на механических примесях, присутствующих в растворе, амины пассивируют активные центры поверхностей, которые могли быть центрами гетерогенной нуклеации [2], что также снижает термодинамическую веро- 
ятность образования зародышей. Незначительное влияние аминов на относительную скорость вторичной нуклеации (см. рис. 2) можно объяснить тем, что основная масса аминов, адсорбируясь на тонкодисперсных частицах $\mathrm{KCl}$ с высокой удельной поверхностью, уже в начале процесса переходит в кристаллическую фазу.

Проведем анализ кристаллизации $\mathrm{KCl}$ по данным распределений частиц. Эволюция дифференциальных кривых числового распределения частиц $\mathrm{KCl}$ по размерам в процессе кристаллизации приведена на рис. 4. На рис. 4 видно, что уже в первые 5 с процесса кристаллизации образуются частицы нескольких типов с доминирующими размерами 22,1 и 74,3 мкм. Причем доля крупных частиц с размерами 74,3 мкм превалирует над мелкими частицами. Наличие в осадке в начальные моменты кристаллизации крупных частиц свидетельствует о том, что рост этих частиц протекал в период накопления в растворе наибольшего пересыщения, когда число частиц и их поверхность были минимальны. Причем, поскольку время роста частиц было очень мало, то рост крупных частиц мог происходить только путем ассоциации мелких частиц, т.е. по агломерационному механизму.

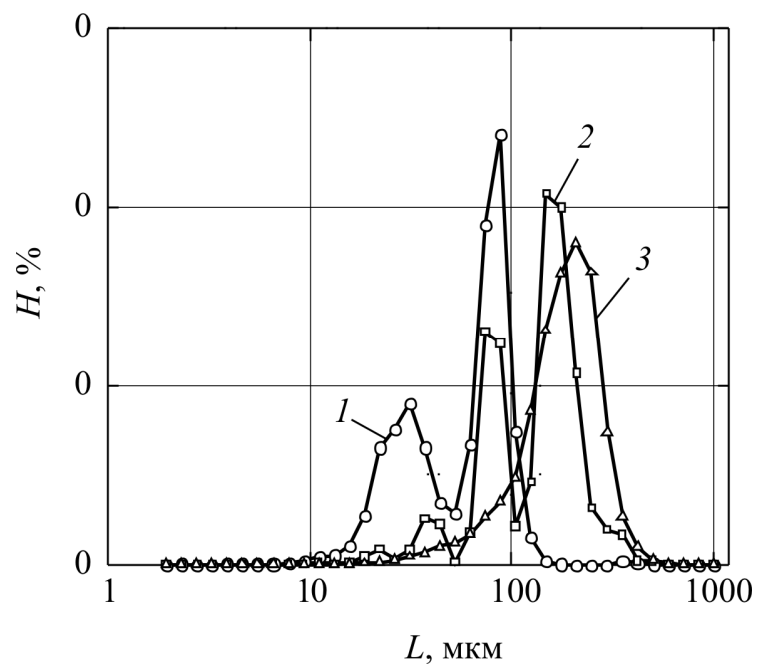

Рис. 4. Дифференциальные кривые числового распределения частиц $\mathrm{KCl}$ по размерам в различные моменты времени процесса политермической кристаллизации: $1-5 \mathrm{c} ; 2-25 \mathrm{c} ; 3-650$ с

Проанализируем динамику изменения содержания фракции класca-11 мкм и содержания крупной фракций (класса +125 мкм), формирование которых в процессе кристаллизации отражает, следовательно, 
вклад процессов генерации частиц и их агломерации в функции распределения (рис. 5). Из анализа кривых рис. 5 следует, что с момента начала массовой кристаллизации в кристаллизуемой среде протекает интенсивное первичное зародышеобразование (максимум на кривых 1,2 ), затем доля мелких частиц падает и начиная со 110 с возрастает. Изменение содержания крупных фракций по мере протекания кристаллизации (кривые 3,4 ) показывает, что агломерационный рост частиц $\mathrm{KCl}$ происходит с наибольшей скоростью на $110 \mathrm{c}$, а затем усиливается механическое разрушение агломератов с образованием тонкодисперсных частиц. Из анализа кривых рис. 5 также видно, что в присутствии 0,001 \% амина почти в 2 раза снижается содержание тонкодисперсных фракций, в то время как содержание крупных агломератов возрастает. Следовательно амины, содержащиеся в чистом растворе в небольших количествах, не блокируют агломерационный рост частиц КСl. Представленные данные также показывают, что на начальных этапах кристаллизации превалирует первичное зародышеобразование, роль которого в образовании тонкодисперсных частиц быстро уменьшается за счет агломерационного роста частиц. В свою очередь роль агломерационного роста частиц по мере кристаллизации постепенно снижается за счет механического разрушения агломератов и вторичного зародышеобразования.

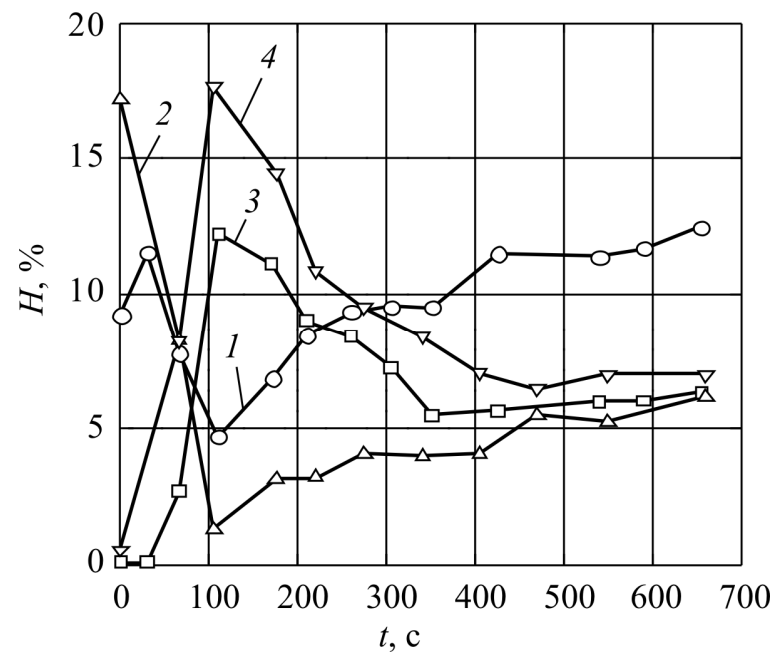

Рис. 5. Изменение числового содержания тонкодисперсной фракции (кривые 1,2 ), и крупной фракции (кривые 3,4 ) частиц $\mathrm{KCl}$ в процессе кристаллизации: 1,3 - из чистого раствора; 2,4 - из раствора, содержащего амин 
Одновременное протекание процессов генерации и формирования частиц в процессе политермической кристаллизации отражается на статистических параметрах образующихся осадков. В таблице приведены статистические характеристики и данные изменения средних размеров частиц $\mathrm{KCl}$ в процессе кристаллизации, вычисленные по методу моментов [10] для функций распределения массы частиц:

$$
L_{4.3}=\alpha \int \mathrm{n}(L) L^{4} d L / n(L) L^{3} d L,
$$

где $\alpha$ - объемный коэффициент формы кристаллов $\mathrm{KCl}$.

\section{Изменение статистических параметров КС1 в процессе массовой} политермической кристаллизации по экспоненциальному режиму охлаждения из чистого раствора и раствора с примесью амина

\begin{tabular}{|c|c|c|c|c|c|c|c|}
\hline $\begin{array}{c}\text { Конц. } \\
\text { амина, } \%\end{array}$ & $t, \mathrm{c}$ & $T,{ }^{\circ} \mathrm{C}$ & $L_{4.3, \text { мкм }}$ & Дисперсия & $\begin{array}{c}\text { Коэф. } \\
\text { вариации }\end{array}$ & $\begin{array}{c}\text { Коэф. } \\
\text { асимметрии }\end{array}$ & Эксцесс \\
\hline 0 & 5 & 70 & 92,7 & 20,37 & 0,219 & 0,175 & 4,573 \\
\hline 0 & 30 & 67 & 86,6 & 25,24 & 0,291 & 0,770 & 5,216 \\
\hline 0 & 65 & 64 & 129,2 & 65,39 & 0,506 & 1,776 & 5,218 \\
\hline 0 & 110 & 60 & 229,0 & 92,35 & 0,403 & 0,363 & 0,120 \\
\hline 0 & 170 & 54 & 224,8 & 90,80 & 0,404 & 0,503 & 0,392 \\
\hline 0 & 210 & 51 & 221,2 & 89,72 & 0,406 & 0,380 & 0,206 \\
\hline 0 & 260 & 47 & 210,5 & 86,72 & 0,412 & 0,553 & 0,854 \\
\hline 0 & 305 & 44 & 206,8 & 86,66 & 0,419 & 0,552 & 0,737 \\
\hline 0 & 350 & 41 & 200,5 & 89,00 & 0,444 & 0,622 & 0,746 \\
\hline 0 & 540 & 32 & 193,8 & 85,88 & 0,443 & 0,694 & 0,997 \\
\hline 0 & 655 & 28 & 199,4 & 86,22 & 0,432 & 0,560 & 0,587 \\
\hline 0,001 & 5 & 68 & 137,6 & 86,69 & 0,630 & 0,213 & $-1,491$ \\
\hline 0,001 & 65 & 64 & 197,8 & 94,13 & 0,476 & 0,863 & 0,972 \\
\hline 0,001 & 105 & 61 & 258,1 & 112,61 & 0,436 & 0,465 & $-0,159$ \\
\hline 0,001 & 175 & 54 & 237,9 & 98,68 & 0,415 & 0,538 & 0,384 \\
\hline 0,001 & 220 & 50 & 230,4 & 95,12 & 0,413 & 0,331 & 0,111 \\
\hline 0,001 & 275 & 46 & 217,4 & 94,47 & 0,435 & 0,627 & 0,696 \\
\hline 0,001 & 340 & 42 & 211,6 & 91,59 & 0,433 & 0,438 & 0,391 \\
\hline 0,001 & 405 & 38 & 212,9 & 94,75 & 0,445 & 0,557 & 0,767 \\
\hline 0,001 & 550 & 32 & 212,6 & 97,30 & 0,458 & 0,464 & 0,137 \\
\hline 0,001 & 660 & 28 & 210,9 & 94,56 & 0,448 & 0,374 & $-0,123$ \\
\hline
\end{tabular}

Данные таблицы показывают, что средние размеры частиц $\mathrm{KCl}$ имеют наибольшие значения через 220-240 с после начала массовой кристаллизации. Причем в присутствии аминов средний размер частиц $\mathrm{KCl}$ имеет 
более высокие значения. Величины средних размеров, приведенные в таблице, с удовлетворительной точностью описываются уравнениями:

$$
\begin{aligned}
& L=64,46+1,413 t-0,0005431 t^{2}-1181,2 \exp (-492,67 / t), \\
& L=127,1+1,405 t-0,0006993 t^{2}-953,89 \exp (-379,35 / t),
\end{aligned}
$$

где уравнение (6) применимо для кристаллизации из чистого раствора, а уравнение (7) используется для кристаллизации КС1 в присутствии $0,001 \%$ амина.

Из приведенных уравнений были получены уравнения, описывающие скорости роста среднего массового размера кристаллов, соответственно для кристаллизации $\mathrm{KCl}$ из чистого раствора:

$$
L=1,413-0,0010862 t-581853,12 \exp (-492,67 / t) t^{-2}
$$

и для кристаллизации из раствора с добавкой амина:

$$
L=1,405-0,001398 t-361858,17 \exp (-379,35 / t) t^{-2} .
$$

Анализ статистических параметров, приведенных в таблице, показывает, что по мере кристаллизации $\mathrm{KCl}$ из чистого раствора дисперсия размеров частиц (а следовательно, и дисперсии скоростей роста), коэффициенты вариации и асимметрии кривых массового распределения частиц по размерам к $t=110$ с быстро возрастают до определенных значений и далее изменяются незначительно. Дисперсии размеров частиц $\mathrm{KCl}$ в присутствии аминов в течение всего процесса кристаллизации имеют более высокие значения, чем в случае кристаллизации из чистого раствора. Отличие коэффициентов вариации размеров частиц, а также коэффициентов асимметрии и значений эксцессов кривых массового распределения по размерам при кристаллизации из чистого и аминированного раствора проявляется в основном на начальных этапах, при длительности массовой кристаллизации порядка 65 c.

\section{Выводы}

1. Исследована кинетика процесса образования частиц $\mathrm{KCl}$ в ходе политермической кристаллизации. Установлен затухающий характер колебаний скорости генерации частиц. Предложены эмпирические уравнения, описывающие кинетику процессов образования и роста частиц хлорида калия при политермической кристаллизации. 
2. По данным эволюции дифференциальных кривых числового распределения частиц по размерам установлено, что в процессе политермической кристаллизации $\mathrm{KCl}$ уже на начальных этапах проявляется значительная роль агломерационного роста частиц. Влияние такого механизма формирования частиц по мере кристаллизации постепенно снижается за счет механического разрушения агломератов и вторичного зародышеобразования.

3. Введение в чистый кристаллизуемый раствор небольших количеств аминов приводит к снижению скорости первичной нуклеации, но не подавляет агломерационный рост частиц, увеличивает дисперсии размеров частиц в начале процесса политермической кристаллизации.

\section{Список литературы}

1. The Kinetics of Industrial Crystallization / J. Nyvlt, O. Sohnel, M. Matuchova, M. Broul. - Praha, 1985. - 320 c.

2. Матусевич Л.Н. Кристаллизация из растворов в химической промышленности. - М.: Химия, 1968. - 340 с.

3. Хамский Е.В. Кристаллизация в химической промышленности. - М.: Химия, 1979. - 343 с.

4. Закономерности образования осадка $\mathrm{Mg}(\mathrm{OH})_{2}$ в системе $\mathrm{MgCl}_{2}-\mathrm{Ca}(\mathrm{OH})_{2}-\mathrm{H}_{2} \mathrm{O}$ в реакторе периодического действия / В.3. Пойлов, Ю. Кодура, Р. Карвот, К. Грамлих, Л.А. Ощепкова // Журнал прикладной химии. - 1993. - Т. 66, вып. 5. - С. 1004-1011.

5. Закономерности образования частиц хлорида магния при массовой политермической кристаллизации / В.З. Пойлов, О.К. Косвинцев, К. Грамлих, Ю. Кодура, Р. Карвот // Журнал прикладной химии. 1997. - Т. 70, вып. 7. - С. 1093-1097.

6. Пойлов В.З., Дементий Л.В. Закономерности выделения карналлита в процессе политермической кристаллизации // Журнал прикладной химии. - 1988. - Вып. 1. - С. 7-10.

7. Пойлов В.З., Головченко Л.В., Рылов В.Л. Особенности формирования кристаллов карналлита при массовой политермической кристаллизации // Crystal Research and Technology. - Berlin, 1989. - C. 97-101.

8. Кристаллизация из растворов по механизму микроблочного роста: теория и моделирование / Г.Г. Филиппов, Л.В. Виленкина, Л.П. Портнов, А.И. Горбунов // Химическая промышленность. - 1993. - № 8. C. $105-110$. 
9. Shmok K. Modelling of mechanism of agglomeration of $\mathrm{KCl}$ crystallization // Summaries 10th Simposium on Industrial Crystallization.Bechine-Castle, CSSR, 1987. - № 154.

10. Randolph F.D., Larson M.A. Theory of Particulate Processes. N.Y.: Academic Press, 1971. -357 p.

\section{References}

1. Nyvlt J., Sohnel O., Matuchova M., Broul M. The Kinetics of Industrial Crystallization. Praha, 1985. 320 p.

2. Matusevich L.N. Kristallizatsiya iz rastvorov $\mathrm{v}$ khimicheskoj promyshlennosti [Crystallization from solutions in chemical industries]. Moscow: Khimiya, 1968. 304 p.

3. Khamskij E.V. Kristallizatsiya v khimicheskoj promyshlennosti [Crystallization in the Chemical Industry]. Moscow: Khimiya, 1979. 343 p.

4. Pojlov V.Z., Kodura Yu., Karvot R., Gramlikh K., Oshchepkova L.A. Zakonomernosti obrazovaniya osadka $\mathrm{Mg}(\mathrm{OH})_{2}$ v sisteme $\mathrm{MgCl}_{2}-\mathrm{Ca}(\mathrm{OH})_{2}-\mathrm{H}_{2} \mathrm{O}$ v reaktore periodicheskogo dejstviya [Formation of $\mathrm{Mg}(\mathrm{OH})_{2}$ precipitation in $\mathrm{MgCl}_{2}-\mathrm{Ca}(\mathrm{OH})_{2}-\mathrm{H}_{2} \mathrm{O}$ system in a batch reactor]. Zhurnal prikladnoj khimii, 1993, vol. 66, iss. 5, pp. 1004-1011.

5. Pojlov V.Z., Kosvintsev O.K., Gramlikh K., Kodura Yu., Karvot R. Zakonomernosti obrazovaniya chastits khlorida magniya pri massovoy politermicheskoy kristallizatsii [Formation of magnesium chloride particles in mass polythermal crystallization]. Zhurnal prikladnoj khimii, 1997, vol. 70, iss. 7, pp. 1093-1097.

6. Pojlov V.Z., Dementij L.V. Zakonomernosti vydeleniya karnallita v protsesse politermicheskoy kristallizatsii [Patterns of carnallite formation during polythermal mass crystallization]. Zhurnal prikladnoj khimii, 1988, iss. 1 , pp. 7-10.

7. Pojlov V.Z., Golovchenko L.V., Rylov V.L. Osobennosti formirovaniya kristallov karnallita pri massovoy politermicheskoy kristallizatsii [Peculiarities of carnallite crystal formation during polythermal mass crystallization]. Crystal Research and Technology. Berlin, 1989, pp. 97-101.

8. Filippov G.G., Vilenkina L.V., Portnov L.P., Gorbunov A.I. Kristallizatsiya iz rastvorov po mekhanizmu mikroblochnogo rosta: teoriya i modelirovanie [Crystallization from solution by the mechanism of microblock growth: Theory and Modeling]. Khimicheskaya promyshlennost', 1993, no. 8, pp. 105-110. 
9. Shmok K. Modelling of mechanism of agglomeration of $\mathrm{KCl}$ crystallization. Summaries 10 th Simposium on Industrial Crystallization. Bechine-Castle, CSSR, 1987, pp. 154.

10. Randolph F.D., Larson M.A. Theory of Particulate Processes. N.Y: Academic Press, 1971. 357 p.

Получено 16.05.2016

\section{Об авторе}

Пойлов Владимир Зотович (Пермь, Россия) - доктор технических наук, профессор, заведующий кафедрой химических технологий Пермского национального исследовательского политехнического университета (614990, г. Пермь, Комсомольский пр., 29, e-mail: vladimirpoilov@mail.ru).

\section{About the author}

Vladimir Z. Pojlov (Perm, Russian Federation) - Doctor of Technical Sciences, Professor, Head of Department of Chemical technology, Perm National Research Polytechnic University (29, Komsomolsky av., Perm, 614990, Russian Federation; e-mail: vladimirpoilov@mail.ru). 